\title{
Psoas muscle area: a new standard for frailty assessment in cardiac surgery?
}

\author{
Leora B. Balsam \\ Division of Cardiac Surgery, UMass Memorial Medical Center, Worcester, MA, USA \\ Correspondence to: Leora B. Balsam, MD. Division of Cardiac Surgery, UMass Memorial Medical Center, University Campus, 55 Lake Avenue North, \\ Worcester, MA 01655, USA. Email: leora.balsam@umassmemorial.org. \\ Provenance: This is an invited Editorial commissioned by the Executive Editor-in-Chief Jianxing He (Department of Cardiothoracic Surgery, The \\ First Affiliated Hospital of Guangzhou Medical University, Guangzhou, China). \\ Comment on: Hawkins RB, Mehaffey JH, Charles EJ, et al. Psoas muscle size predicts risk-adjusted outcomes after surgical aortic valve replacement. \\ Ann Thorac Surg 2018;106:39-45.
}

Submitted Oct 15, 2018. Accepted for publication Oct 23, 2018.

doi: $10.21037 /$ jtd.2018.10.96

View this article at: http://dx.doi.org/10.21037/jtd.2018.10.96

Most would agree that a successful cardiac operation ends with a live patient who is able to return to a functional and productive life. When evaluating candidates for surgery, a risk/benefit analysis must be performed that takes into account the likely short- and long-term outcomes for the patient. A discussion of these outcomes will become part of the informed consent conversation when surgery is offered. But in some cases, the question is not "Can surgery be offered?" but instead, "Should surgery be offered?" Most patients and their families will hold on to that glimmer of hope that with surgery, they will beat the odds. As clinicians, we are faced with the ethical and moral dilemma of balancing their expectations and using our limited resources judiciously.

Discussions regarding futility have increased as the population undergoing cardiac surgery has become older. Presently, more than half of patients undergoing cardiac surgery are over the age of 75 . Many centers have shown excellent outcomes with both primary and reoperative cardiac surgery in the elderly, but it is clear that patient selection is a major driver of outcomes. High risk features, including end-organ dysfunction, shock, and emergency surgery are all associated with increased surgical risk. Two commonly used risk calculators, the Society of Thoracic Surgeons Predicted Risk of Mortality (STS-PROM) and the European System for Cardiac Operative Risk Evaluation (EuroSCORE) II incorporate demographic and physiologic elements to estimate operative mortality and morbidity, and both are commonly used to guide decision-making in cardiac surgery. At times, neither score captures what is obvious to the clinician at the bedside with 'the eyeball test'. Looking at the patient, the clinician may instinctively know whether that patient will survive and/or thrive after surgery. This gut feeling bears some influence on surgical decisionmaking, and we often call it clinical judgment.

The eyeball test acts at least in part as a surrogate for frailty testing. Frailty is a syndrome of impaired physiologic reserve and decreased resistance to internal and external stressors, and both aging and chronic diseases result in frailty. Frailty has been associated with a greater risk of adverse postoperative outcomes after a variety of surgical procedures, including vascular, abdominal, and cardiac surgery. With the growing use of transcatheter valve procedures, particularly transcatheter aortic valve replacement (TAVR), in the very elderly and/or high-risk patients, discussions regarding frailty and futility have come to center stage (1-3).

Frailty is difficult to define, though clinicians often say they know it when they see it. As it turns out, there is significant interobserver variability with 'the eyeball test' (4), so objective measures of frailty are needed. Numerous frailty tool-sets have been developed and validated in different populations, including cardiac surgical patients. These tests include multidimensional scales that assess muscle weakness, malnutrition, cognitive impairment, functional performance, social support, general health status, and mood. Depending on which tool is used, the incidence of frailty in cardiac surgical populations ranges from $25-50 \%$. 
The STS has recognized the importance of frailty in cardiac surgery outcomes, and in 2011 added preoperative 5-meter gait speed as a variable in the adult cardiac surgery database. Afilalo $e t a l$. validated the association between gait speed and operative mortality in patients $\geq 60$ years in the STS data-set (5), and several groups have shown that frailty assessment adds incremental value above STS-PROM and EuroSCORE II for predicting adverse early outcomes (6-12). These include mortality, major morbidity, prolonged length of stay, and discharge to a facility rather than home.

The STS-PROM and EuroSCORE II were developed for prediction of early adverse outcomes after cardiac surgery, rather than mid- and long-term outcomes. Frailty assessments may be useful in predicting later outcomes. In the TAVR population, frailty has been associated with poor mid-term outcomes, defined as death or poor health-related quality of life at 1 year. A sub-study of the PARTNER trial reported that at 3 high-enrolling sites where frailty was assessed, all-cause mortality was $32.7 \%$ in the frail group and $15.9 \%$ in the non-frail group (3). At 1 year, poor outcome occurred in $50.0 \%$ of the frail group and $31.5 \%$ of the non-frail group. Several single center studies have reported an association between frailty and worse longterm outcomes after cardiac surgery. Afilalo et al. (13) found that in frail older adults undergoing either TAVR or surgical aortic valve replacement (SAVR), the incidence of death or worsening disability was $50 \%$ at 1 year, compared to an incidence of $35 \%$ in all-comers. A large multicenter European registry reported that frailty increased midterm mortality after coronary artery bypass grafting (CABG), independent of other common risk factors (11). Lytwyn and colleagues also found that frail patients had 2-3.5 times the odds of poor functional survival at 1 year after cardiac surgery in a single center study (14).

Although there is general agreement that identification of frailty is important in cardiac surgical patients, there is no consensus as to which assessment measure(s) for frailty should be used. Some studies suggest that a multidimensional frailty tool is more sensitive, however there are challenges with using these tools in preoperative cardiac surgery patients. The tests may be time-consuming, require specialized equipment, or they may not be possible to perform in the hospital setting. A different approach to quantifying frailty focuses on sarcopenia, an age-related loss of skeletal muscle that is present in $5-10 \%$ of individuals over that age of 65 . Sarcopenia is one element in the frailty syndrome that is unaffected by acute changes in clinical status (for example, hospitalization) or patient effort. A validated method for quantifying sarcopenia is measurement of the psoas muscle area. The psoas muscle, a flexor of the lower extremity and stabilizer of the lumbar vertebral column and hip joint, is involved in walking, standing from a seated position, and maintaining balance. As such, the psoas muscle bears impact on functional recovery after major surgery. Psoas muscle area can be quantified using cross-sectional computed tomography (CT) scans with good inter-observer correlation, and psoas muscle area correlates with sarcopenia. The cut-off value for dividing populations into sarcopenic and non-sarcopenic groups is somewhat arbitrary and based on comparison to sex-specific patterns. In some studies, patients with psoas muscle area in the lowest quartile are considered sarcopenic, and in others, those 2 standard deviations below a mean reference value from healthy young adults are considered sarcopenic. Another approach is to index psoas muscle area to body surface area and treat it as a continuous variable. Psoas muscle area is higher in men than women, and its size decreases with increasing age.

A recent study by Hawkins et al. (15) evaluated the predictive value of psoas muscle size for adverse events after surgical aortic valve replacement in a moderate to high risk group of patients, defined as STS-PROM $>3 \%$. The study was performed at a single center between 2009-2016, and included 240 patients undergoing either isolated SAVR or combined coronary revascularization and SAVR. Patients were excluded from analysis if preoperative CT scans were not available. Psoas muscle area was measured on crosssectional CT images in a standardized manner, and the authors defined sarcopenia as psoas muscle area less than the sex-specific 25 th percentile. In this series, the median patient age was 80 years and the average STS-PROM was $6 \%$. Sarcopenia was present in $33.3 \%$ of patients. Patients with sarcopenia had higher 1 year mortality (31.9\% vs. $16.9 \%$ ). Psoas muscle index (psoas muscle area indexed to body surface area) was predictive of risk-adjusted 1 year mortality, postoperative adverse events including prolonged ventilation, longer hospital length of stay, higher hospital cost, and discharge to a facility. In this particular study, psoas index was not predictive of operative mortality or readmission.

Other groups have also found that psoas muscle area is predictive of outcomes after various cardiac surgical procedures. In a single center study of patients undergoing both surgical aortic valve replacement and TAVR, Paknikar et al. found that lower psoas muscle area was an independent predictor of 30-day mortality, high resource utilization, and late mortality (8). The association with early 
mortality is notably different than that reported by Hawkins et al. (15). Zuckerman et al. reported that lower psoas muscle area predicted increased length of stay after cardiac surgery, with a 2-day increase in length of stay for every $1 \mathrm{~cm}^{2}$ decrement in psoas muscle area (9). Okamura et al. also found that sarcopenic patients had decreased freedom from major adverse cardiac and cerebrovascular events after heart valve surgery in a large single center study (7). Moreover, sarcopenia was also an independent predictor of decreased long-term survival in their multivariable analysis. In cardiac transplant patients, lower psoas muscle area is also associated with increased in-hospital mortality or major morbidity and worse long-term survival (16).

The advantages of using psoas muscle area as a frailty measure have been described above, however the disadvantages are worth discussing. First, it requires crosssectional imaging, which has an associated cost. In the present era, a growing number of elderly and moderate to high risk patients are undergoing preoperative CT evaluation as part of the decision-making process for TAVR versus SAVR, as well as other cardiac surgeries. This CT evaluation allows for identification of hostile aortic anatomy that may affect treatment choice and/or surgical risk. Second, it is not clear that psoas muscle area is the most sensitive or specific for frailty, and a more comprehensive frailty tool-set may have advantages in that respect.

It is still relatively unknown which procedures will lead to reversal of frailty and under which conditions. More investigation is needed in this area. One may ask, are there specific cut-off values for the psoas muscle index where reversal is no longer possible? Moreover, is frailty that is identified by the psoas muscle index actionable? Will physical rehabilitation prior to surgery (prehabilitation) be effective in a certain subset of patients? If so, which ones? While it is clear that frailty influences outcomes after cardiac surgery, major questions remain. Identifying which components of the frailty assessment are most predictive of adverse outcomes and incorporating these into a standard preoperative evaluation and/or risk score are important future directions.

\section{Acknowledgements}

None.

\section{Footnote}

Conflicts of Interest: The author has no conflicts of interest to declare.

\section{References}

1. Forcillo J, Condado JF, Ko YA, et al. Assessment of Commonly Used Frailty Markers for High- and ExtremeRisk Patients Undergoing Transcatheter Aortic Valve Replacement. Ann Thorac Surg 2017;104:1939-46.

2. Arnold SV, Afilalo J, Spertus JA, et al. Prediction of Poor Outcome After Transcatheter Aortic Valve Replacement. J Am Coll Cardiol 2016;68:1868-77.

3. Green P, Arnold SV, Cohen DJ, et al. Relation of frailty to outcomes after transcatheter aortic valve replacement (from the PARTNER trial). Am J Cardiol 2015;116:264-9.

4. Yanagawa B, Graham MM, Afilalo J, et al. Frailty as a risk predictor in cardiac surgery: Beyond the eyeball test. J Thorac Cardiovasc Surg 2018;156:172-6.e2.

5. Afilalo J, Kim S, O'Brien S, et al. Gait speed and operative mortality in older adults following cardiac surgery. JAMA Cardiol 2016;1:314-21.

6. Amabili P, Wozolek A, Noirot I, et al. The Edmonton Frail Scale Improves the Prediction of 30-Day Mortality in Elderly Patients Undergoing Cardiac Surgery: A Prospective Observational Study. J Cardiothorac Vasc Anesth 2018. [Epub ahead of print].

7. Okamura H, Kimura N, Tanno K, et al. The impact of preoperative sarcopenia, defined based on psoas muscle area, on long-term outcomes of heart valve surgery. J Thorac Cardiovasc Surg 2018. [Epub ahead of print].

8. Paknikar R, Friedman J, Cron D, et al. Psoas muscle size as a frailty measure for open and transcatheter aortic valve replacement. J Thorac Cardiovasc Surg 2016;151:745-51.

9. Zuckerman J, Ades M, Mullie L, et al. Psoas muscle area and length of stay in older adults undergoing cardiac operations. Ann Thorac Surg 2017;103:1498-504.

10. Bergquist CS, Jackson EA, Thompson MP, et al. Understanding the Association Between Frailty and Cardiac Surgical Outcomes. Ann Thorac Surg 2018. Ann Thorac Surg 2018;106:1326-32.

11. Reichart D, Rosato S, Nammas W, et al. Clinical frailty scale and outcome after coronary artery bypass grafting. Eur J Cardiothorac Surg 2018. Eur J Cardiothorac Surg 2018;54:1102-9.

12. Sepehri A, Beggs T, Hassan A, et al. The impact of frailty on outcomes after cardiac surgery: a systematic review. J Thorac Cardiovasc Surg 2014;148:3110-7.

13. Afilalo J, Lauck S, Kim DH, et al. Frailty in older adults undergoing aortic valve replacement: the FRAILTY-AVR 
Study. J Am Coll Cardiol 2017;70:689-700.

14. Lytwyn J, Stammers AN, Kehler DS, et al. The impact of frailty on functional survival in patients 1 year after cardiac surgery. J Thorac Cardiovasc Surg 2017;154:1990-9.

15. Hawkins RB, Mehaffey JH, Charles EJ, et al. Psoas muscle

Cite this article as: Balsam LB. Psoas muscle area: a new standard for frailty assessment in cardiac surgery? J Thorac Dis 2018;10(Suppl 33):S3846-S3849. doi: 10.21037/jtd.2018.10.96 size predicts risk-adjusted outcomes after surgical aortic valve replacement. Ann Thorac Surg 2018;106:39-45.

16. Bibas L, Saleh E, Al-Kharji S, et al. Muscle Mass and Mortality After Cardiac Transplantation. Transplantation 2018. [Epub ahead of print]. 